\title{
Improving Process Quality by Means of Accurate and Traceable Calibration of Flow Devices with Process-oriented Liquids
}

\author{
Hugo Bissig*, Martin Tschannen, and Marc de Huu
}

\begin{abstract}
Calibration of flow devices is important in several areas of pharmaceutical, flow chemistry and health care applications where volumetric dosage or delivery at given flow rates are crucial for the process. Although most of the flow devices are measuring flow rates of process-oriented liquids, their calibrations are often performed with water as calibration liquid. It is recommended to perform the calibrations of the flow devices with process-oriented liquids as the liquid itself might influence the performance of the flow devices. Therefore, METAS has developed facilities with METAS flow generators to address the issue of measuring with processoriented liquids for flow rates from $400 \mathrm{ml} / \mathrm{min}$ down to $50 \mathrm{nl} / \mathrm{min}$ with uncertainties from $0.07-0.9 \%$. Traceability is guaranteed through the calibration of the generated flow rates of the METAS flow generators by means of the dynamic gravimetric method where a liquid of well-known density and a well-controlled evaporation rate is used. The design of the milli-flow facility will be discussed as well as first measurement results of the METAS flow generators in the range of micro-flow and milli-flow using water and other liquids.
\end{abstract}

Keywords: Dynamic gravimetric calibration · Flow generator · Microfluidics · Process liquids · Traceability

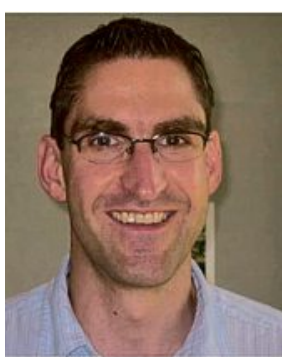

\section{Introduction}

Calibration of flow devices is important in several areas of pharmaceutical, flow chemistry and health care applications where volumetric dosage or delivery at given flow rates are crucial for the process. Although most of the flow devices are measuring flow rates of process-oriented liquids, their calibrations are often performed with water as calibration liquid. It is recommended to perform the calibrations of the flow devices with process-oriented liquids as the liquid itself might influence the performance of the flow devices.

After developing a facility for the micro-flow range, ${ }^{[1,2]}$ METAS has developed a facility with METAS flow generators to address the issue of measuring with process-oriented liquids and to extend its international traceability for flow rates up to $400 \mathrm{ml} / \mathrm{min}$ with an uncertainty of $0.07 \%$. The METAS flow generators are homemade syringe pumps which allow measurements with liquids other than water in the range from $400 \mathrm{ml} / \mathrm{min}$ down to $50 \mathrm{nl} /$ min. Traceability is guaranteed through the calibration of the generated flow rates of the METAS syringe pumps by means of the dynamic gravimetric method where a liquid of well-known density and a wellcontrolled evaporation rate is used. As the METAS syringe pump is a volumetric flow generator, it can be operated with any liquid acting as a transfer standard to perform calibrations of flow devices. The ad- vantage of traceable calibrations of a flow device with the process-oriented liquid is to enhance the quality of the measurement results of the flow device during the production process. Different types of flow devices are stated from the manufacturer with accuracies between $0.5 \%$ and $10 \%$ dependent on the flow rate range and the working principle of the flow devices. Obviously, a traceable calibration with uncertainties ranging from $0.07 \%$ to $0.9 \%$ for flow rates ranging from $400 \mathrm{ml} / \mathrm{min}$ to $50 \mathrm{nl} / \mathrm{min}$ will enhance the accuracy of the measurements as the stated deviations of the flow devices are corrected in the application and the measurement uncertainties are known and much smaller than the accuracies stated by the manufacturer. As an example we show in Fig. 1 the deviations with respect to the reference flow rate of two flow devices of the same type (blue and green symbols) obtained from calibration. The stated accuracy of the manufacturer is $5.0 \%$ (red line). Moreover, calibration data over a long period of time allow the reproducibility and drift of the flow device to be determined and included in the measurement uncertainty. For simplicity, in some cases the deviations as a function of the flow rate can be described by a linearized function. Therefore, the uncertainty for each measurement point would slightly be increased, but the application of the linearized function for the correction of the measured deviation would be easier. In this example, the linearized deviation is shown 


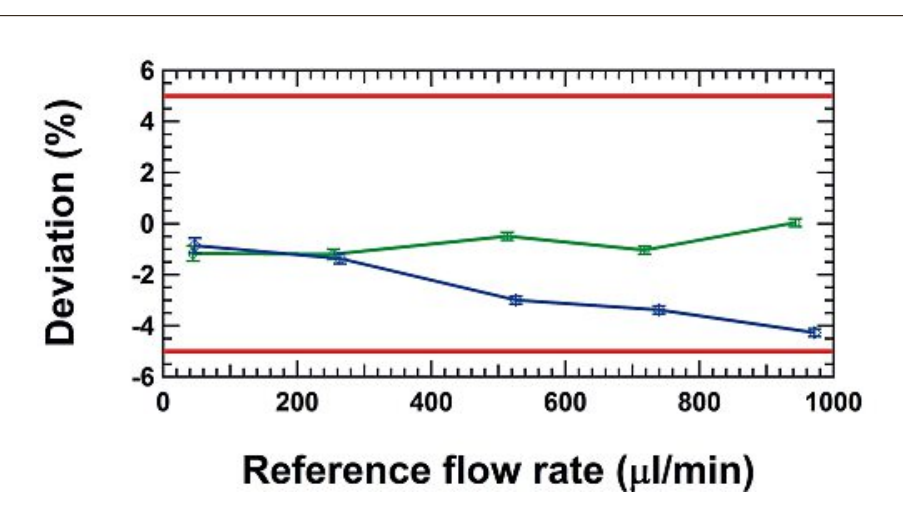

Fig. 1. Deviations of two flow devices of the same type (blue and green symbols) obtained after calibration. The stated accuracy of the manufacturer is $5.0 \%$ (red line).

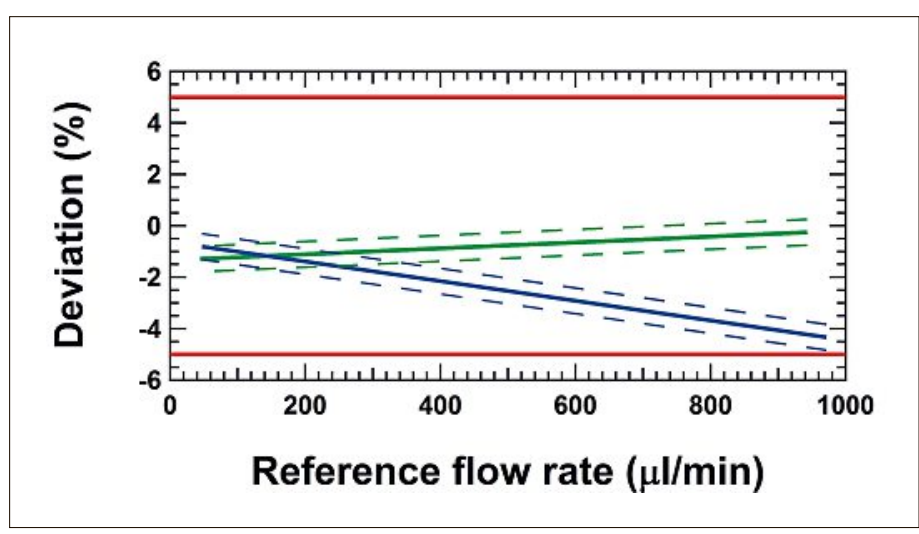

Fig. 2. Deviations of two flow devices of the same type (blue and green lines) obtained after calibration and linearized function of the deviations. The final measurement uncertainty is $0.5 \%$ (indicated by the dashed blue and green lines). The stated accuracy of the manufacturer is $5.0 \%$ (red line). as blue and green solid line in Fig. 2 with a final measurement uncertainty of $0.5 \%$ (indicated by the dashed blue and green lines). This case shows the advantage of the calibration by obtaining a final uncertainty which is 10 times better than the stated accuracy of the flow device. It is important to state that the deviations obtained by the calibration are within the accuracy stated by the manufacturer, but there will be an enhancement of the performance of the flow device for the process application.

Currently, validated traceability in the micro-flow range in Europe goes down to flow rates of $100 \mathrm{nl} / \mathrm{min}$. [3] Therefore, traceable calibrations in Europe are guaranteed for flow rates of several orders of magnitude up to several thousands of $\mathrm{m}^{3} / \mathrm{h}$. Nevertheless, most of the calibration facilities are operated with water and are not designed to be operated with any other liquid than water. A minority of laboratories offers the calibration also for oil products. [4-6]

\section{Design of the Milli-flow Facility}

The milli-flow facility is designed to cover the flow rate range from $0.2 \mathrm{ml} / \mathrm{min}$ up to $400 \mathrm{ml} / \mathrm{min}$ with an uncertainty of $0.07 \%$ (coverage factor $95 \%$ ).

\subsection{Working Principle}

The METAS flow generator, a homemade syringe pump, is filled with water and the water is pressed at the desired flow rate through the DUT (device under test) and collected in the beaker on the balance, as shown in Fig. 3. More details can be seen in Fig. 4, where a photo of the actual facility is shown.

The installation is filled with degassed ultrapure water. To hamper growth of bacteria and algae, $50 \mathrm{mg}$ of sodium azide is mixed with 2 liters of ultrapure water, which is not hazardous for the environment in this concentration and does not affect the density determination at the requested uncertainty.

An important issue is to avoid any air entrapment each time a DUT is connected to the facility. The calibration procedure has to make sure that the air inside the DUT is flushed out. Close to the connections of the DUT, a purging system with $\mathrm{CO}_{2}$ is installed, where the DUT is first flushed with $\mathrm{CO}_{2}$ and then flushed with water, where the $\mathrm{CO}_{2}$ and the water are directed to the piping for waste. Thus, the DUT is full of water and no air remains in the piping.

\subsection{METAS Flow Generator}

The METAS flow generator consists of a high precision linear stage with a fixed linear measuring system, mounting parts to fix syringes in front of the table and mounting parts to fix and move the plunger of the syringe in order to generate the flow rate (see Fig. 5). The position of the linear stage is determined by counting the pulses sent by the linear measuring system by means of an FPGA, which is a Field Programmable Gate Array with hard coded program code running on a defined constant cycle time of the order of $25 \mathrm{~ns}$. For each additional pulse in any direction, a time stamp of the FPGA is recorded and a pair with the position and the timestamp is formed. This pair of values is then read from the main software and the real time position can be recorded. The real time speed is then determined by a linear fit of the position as a function of time. Multiplying the speed with the cross section of the syringe gives the volume flow rate.

\subsection{Evaporation and Continuous Water Bridge}

To limit the contribution of water evaporation to the measurement, the evaporation rate in the beaker has to be kept low during the measurement. To control this process, the conventional method of adding water in an evaporation trap in the weighing zone (blue colored water in Fig. 6D) to saturate the air with humidity is applied. To avoid any condensation on the outlet needle the degree of humidity is regulated by two small holes at the top of the weighing zone housing, which are connected to tubing acting as humidity exchanger (Fig. 6G).

Additionally, a special measurement beaker is built, as shown in the detailed cross-section in Fig. 6B. The outlet needle is in general positioned $200 \mu \mathrm{m}$ to $50 \mu \mathrm{m}$ above the glass filter (Fig. 6C) depending on the diameter of the outlet needle $(1 \mathrm{~mm}$ to $0.3 \mathrm{~mm}$ ). At low flow rates, the water enters the measurement beaker and the capillary force in the glass filters sucks the water in before any droplet can be formed at the surface. The water finds its way through the glass filter and continues

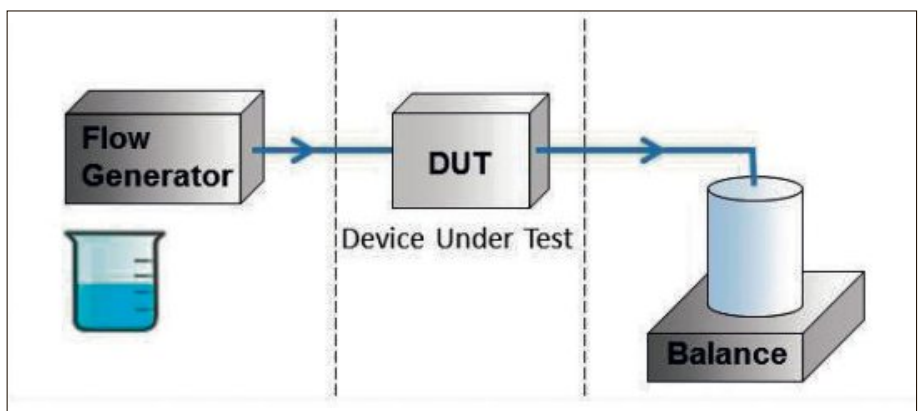

Fig. 3. Working principle of the milli-flow facility. The flow generator presses the water through the DUT in the beaker on the balance, where it is collected. 


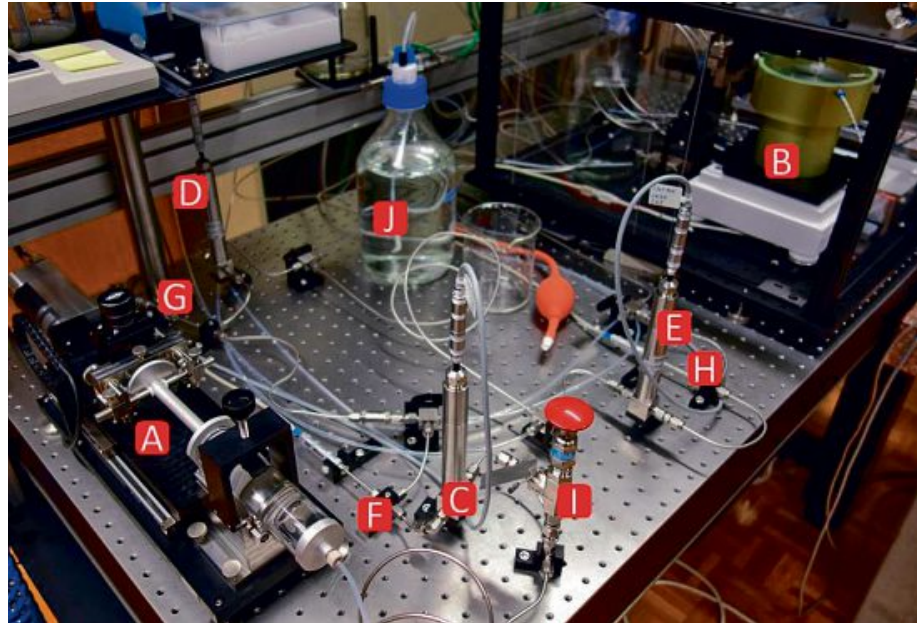

Fig. 4. Milli-flow facility. (A) METAS syringe pump, (B) beaker on balance, $(C, D, E)$ pressure sensors, $(F, G, H)$ temperature sensors, (I) pressure security valve, $(J)$ water reservoir.

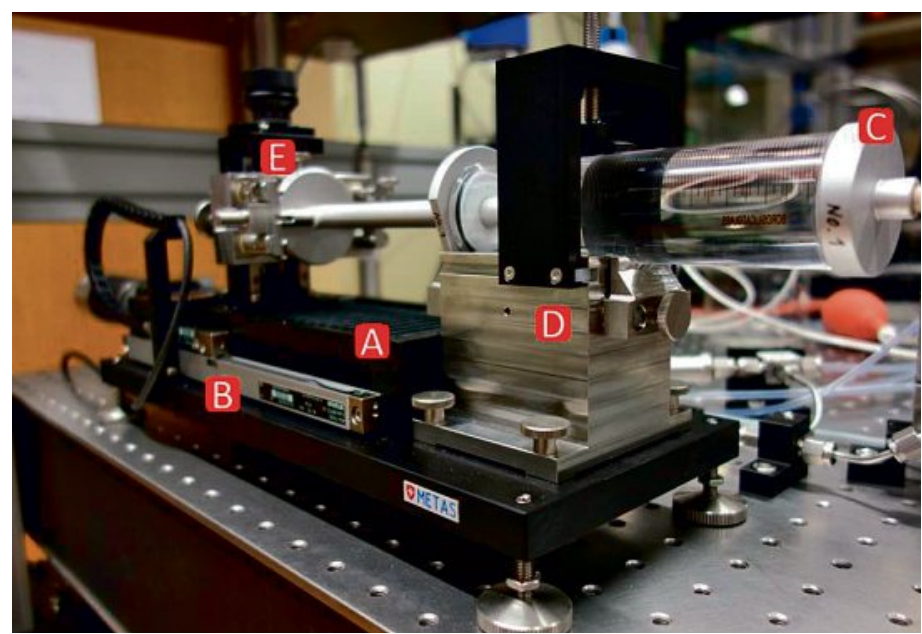

Fig. 5. METAS flow generator. (A) high precision linear stage, (B) linear measuring system, (C) syringe, (D) mounting syringe body, $(E)$ mounting and positioning for syringe plunger. in the bulk of water at the bottom of the beaker. If the flow rate is higher, the water jet hits the surface of the glass filter and the water flows down at the surface of the glass filter. In both cases the forces due to the water bridge are constant in time and they do not influence the measurement. With this setup, a stable evaporation rate of $(3.5 \pm 0.5) \mathrm{nl} / \mathrm{min}$ for water is achieved and hardly contributes to the uncertainty.

\section{Dynamic Gravimetric Method}

The measurements are performed by means of the dynamic gravimetric flying start-stop method. This means that the desired flow rate is set and the data acquisition is only started once the flow rate has reached a steady state. Therefore, the measurement beaker is continuously filled with water and the weighing data are continuously collected by a Real Time system (RT), which communicates with the balance at $10 \mathrm{~Hz}$. The weight value is directly paired with the time stamp of the RT. The other sensor values such as water pressure upstream and downstream of the DUT, the water temperature at various positions and the ambient conditions are recorded as well.

\subsection{Determination of Flow Rate}

The collected weighing data are then fitted by means of a least square linear fit. Flow rate determination is best explained using an example. A fixed time window of 10 seconds is chosen for the determination of the instantaneous flow rate, shown in Fig. 7 as green and orange portions on the black curve showing the weighing value. The resulting mass flow rates are then converted to volume flow rates including all corrections (evaporation, buoyancy, etc.) and are shown in Fig. 8 as green and or- ange triangles with their uncertainties. The time stamp of the determined volume flow rate of each fixed time window is the center time of these data. This guarantees that any strong change in the flow rate is detected at the occurring time independently of the chosen fixed time window. By increasing the starting time of this fixed time window by time steps corresponding to the acquisition rate of $10 \mathrm{~Hz}$ the moving average of the flow rate in time can be followed. Applying this to the collected weighing data shown in Fig. 7 (black line), the evolution in time of the volume flow rate is obtained, as shown in Fig. 8 (black line).

The same procedure applies to the data of the linear measuring system, where the identical fixed time window is chosen. The change of the position in time shown in Fig. 7 (red line) leads to the evolution in the flow rate shown in Fig. 8 (red line), where the speed of the syringe pump is multiplied by the cross section of the syringe to obtain the volume flow rate. The cross section is the nominal value from the manufacturer's datasheet. The background in gray in Fig. 8 illustrates the time window, where the flow rate is considered to be in steady state after a stabilization time. Once the stabilization time is reached, the deviation of the flow rate of the syringe pump with respect to the flow rate determined by the dynamic gravimetric method can be calculated. Otherwise a systematic error on the deviation can be introduced, which is only dependent on the stabilization of the flow rate of the facility and not on the DUT itself.

\subsection{Buoyancy Correction Online}

Another issue of the dynamic measurement is the continuous filling of the measurement beaker with water. ${ }^{[1]}$ Therefore the buoyancy correction $f_{\text {buoyancy }}$ for the increasing water volume in the measurement beaker depends on the evolution over time of the air density and the water density in the beaker. To take any unforeseen changes in the densities into account, the buoyancy correction factor is calculated for each single fixed fit window and directly applied to the mass flow rate $Q_{w}$ obtained from the real weight values (not the conventional

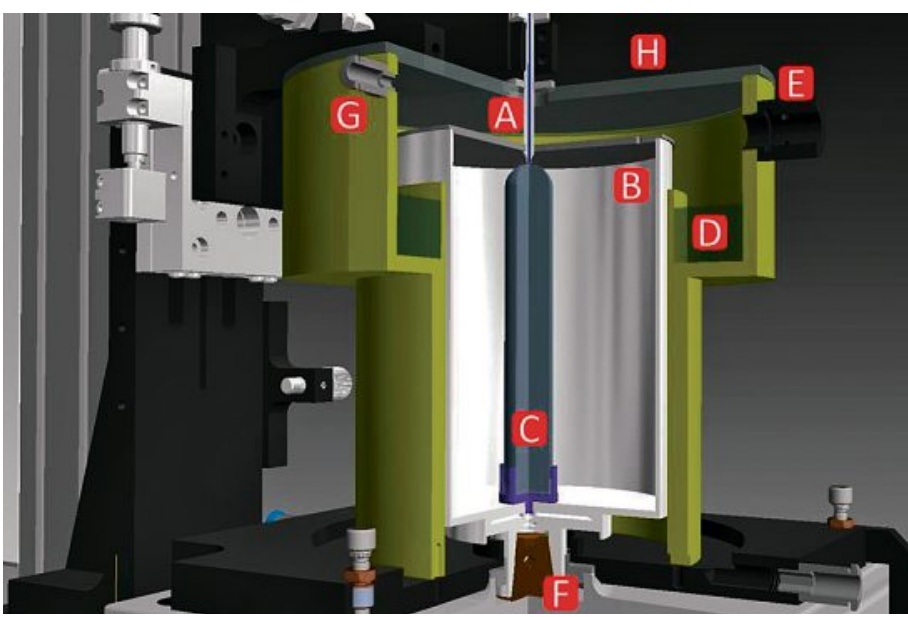

Fig. 6. Weighing zone on the balance. (A) outlet needle, (B) beaker with cover, (C) glass filter, (D) water in evaporation trap, (E) mount for $\mathrm{T}$ and $\mathrm{rH}$ sensor, (F) balance,

(G) tubing for humidity exchanger, $(\mathrm{H})$ cover of the weighing zone. 


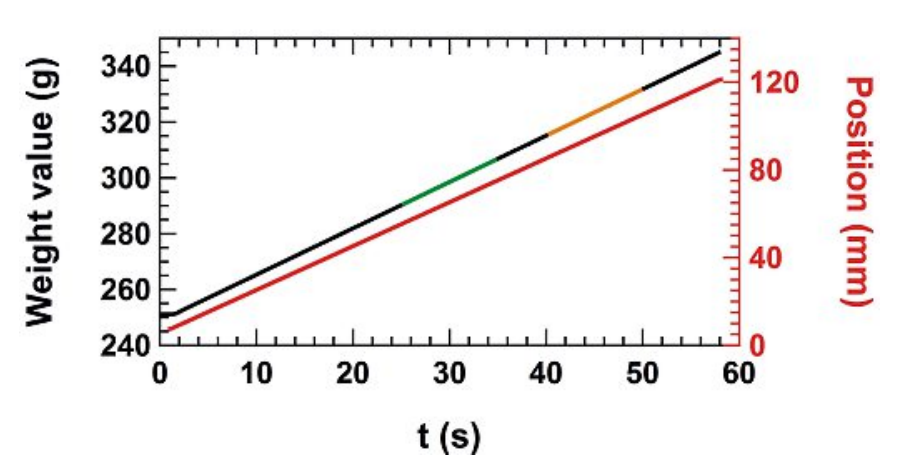

Fig. 7. Increase of mass on the balance (black line) and increase of the position of the linear measuring system (red line) as a function of time. Two fixed time windows of 10 seconds are shown in green and orange on the black line.

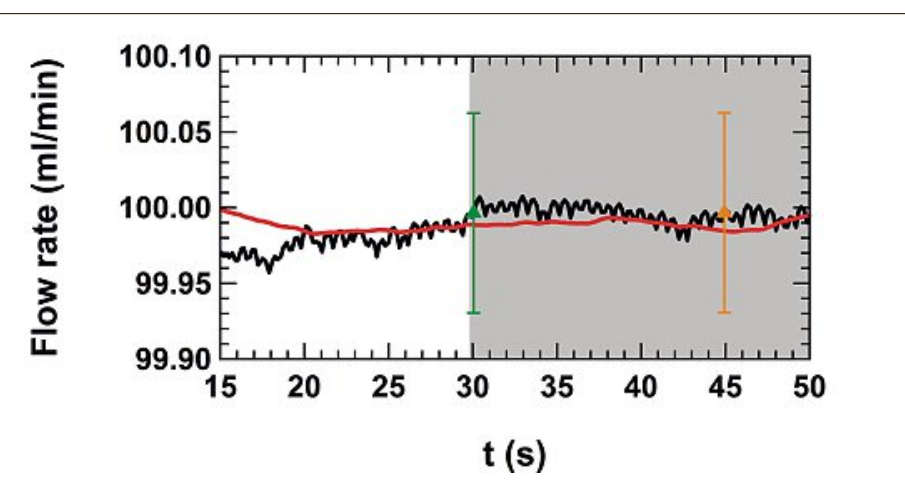

Fig. 8. The determined volume flow rate of the balance (black line) and of the syringe pump (red line) as a function of time. The green and the orange triangles are the determined volume flow rates corresponding to the data in the fixed time windows (green and orange) in Fig. 7. The background in gray illustrates the time window where the flow rate is considered to be in steady state after the stabilization time. weighing values), as shown in the simplified Eqn. (1).

$$
Q_{m}(t)=\left(Q_{w}(t) \cdot f_{\text {buoyancy }}(t)-\dot{m}_{\text {evap }}\right)
$$

$\dot{m}_{\text {evap }}$ is the evaporation rate of the water from the beaker on the balance.

\section{Repeatability and Reproducibility of the Milli-flow Facility}

In Fig. 9 and Fig. 10, first measurement results with the milli-flow syringe pump using a syringe of $100 \mathrm{ml}$ and $10 \mathrm{ml}$ volume respectively, are shown. Symbols with the same color represent the repeatability measurements where the syringe was mounted and calibrated several times. Symbols with different color represent the reproducibility measurements as the syringe was mounted for a first calibration and then removed from the mounting block and remounted several days later for a second calibration. The repeatability and the reproducibility measurements are consistent and prove the suitability for changing the syringes on the mounting in order to change the flow rate range, which is limited to the speed range of the linear stage and the diameter of the syringe.

The mean values of the three calibrations for each flow rate with the two different syringes are shown in Fig. 11. The consistent deviations over the full flow rate range for each syringe mounted on the syringe pump indicate that several calibration points are sufficient for the calibration of the corresponding syringe. The average of the deviations leads to a single calibration factor of the syringe for the full flow rate range.

\section{Repeatability of the Micro-flow Flow Generator}

For flow rates below $0.2 \mathrm{ml} / \mathrm{min}$, METAS has built the same flow generator with a lower speed range called the microflow flow generator to cover the flow rate range from $100 \mathrm{nl} / \mathrm{min}$ to $5 \mathrm{ml} / \mathrm{min}$, which is already covered by the micro-flow fa- cility. ${ }^{[1]}$ The first measurements with the micro-flow flow generator using syringes of $50 \mathrm{ml}, 10 \mathrm{ml}$ and $1 \mathrm{ml}$ on the micro-flow facility are shown in Fig. 12. Rather consistent deviations are found over the full flow rate range for each syringe mounted on the syringe pump. Also in the case of this micro-flow syringe pump the average of the deviations leads to a single calibration factor of the syringe for the full flow rate range.

\section{Calibration Using Reference Oils}

As the volume flow rates of the milliflow flow generator are now calibrated with water and traceable to the SI units, it can be operated with any other liquid. In order to confirm this, additional calibrations are performed using three reference oils with traceable densities and viscosities of $16 \mathrm{mPa} \mathrm{s}$, $33 \mathrm{mPa}$ and $120 \mathrm{mPa}$ at $22^{\circ} \mathrm{C}$ by means of the dynamic gravimetric method, as shown in Fig. 13. Due to the higher viscosities of the oils, the pressure drop in the piping and in the DUT are much higher compared to

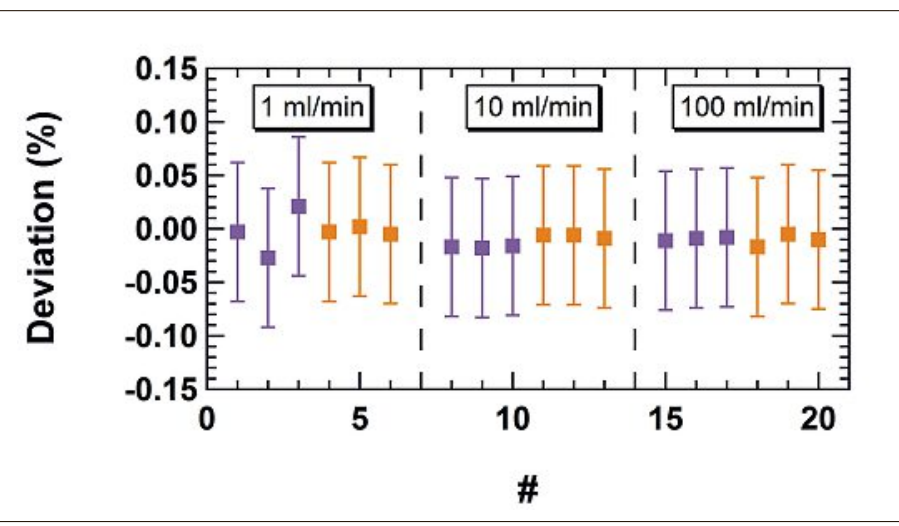

Fig. 9. Calibration of the milli-flow flow generator using a $100 \mathrm{ml}$ syringe. The purple and orange squares represent repeatability measurements whereas the different color codes represent reproducibility measurements.

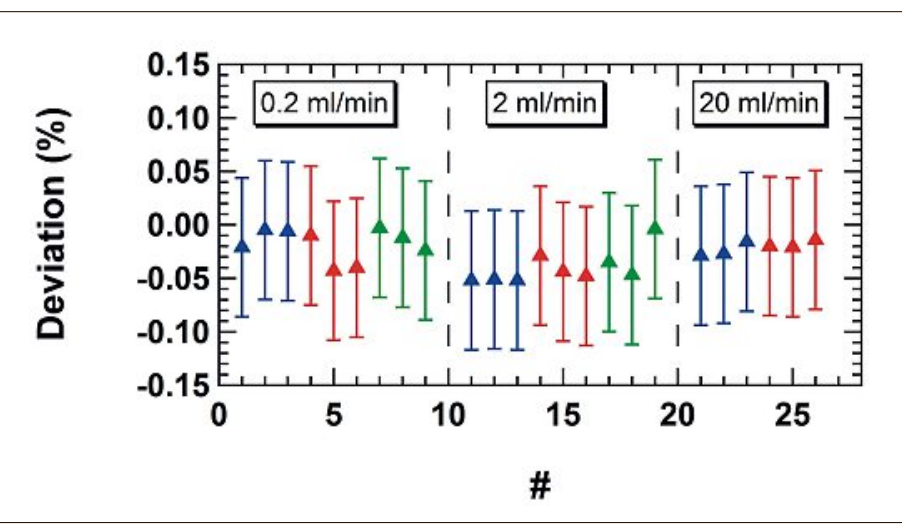

Fig. 10. Calibration of the milli-flow flow generator using a $10 \mathrm{ml}$ syringe. The blue, red and green triangles represent repeatability measurements whereas the different color codes represent reproducibility measurements. 


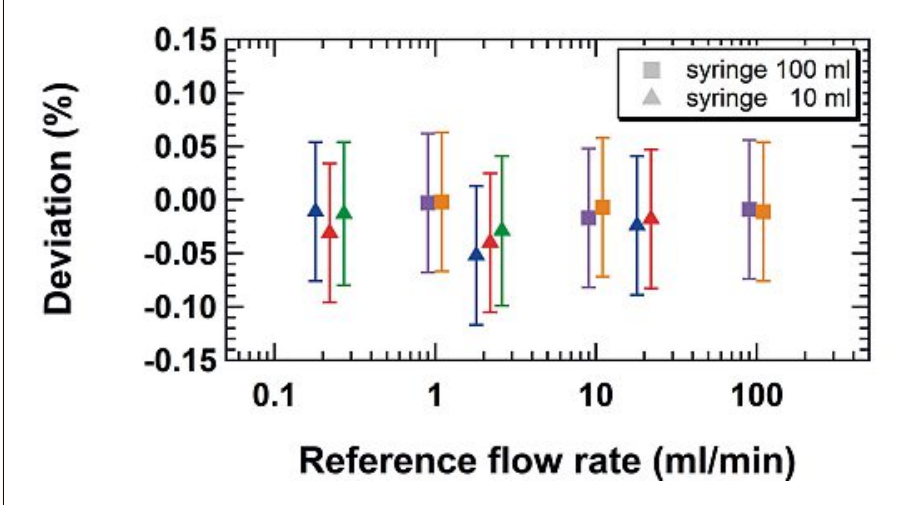

Fig. 11. Mean values of the deviations shown in Figs 9 and 10 for the syringes of $100 \mathrm{ml}$ (colored squares) and $10 \mathrm{ml}$ volume (colored triangles).

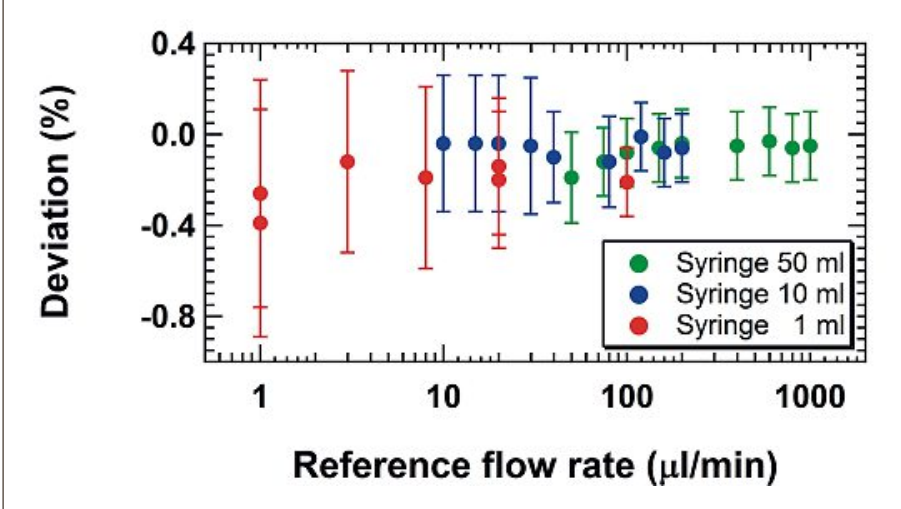

Fig. 12. First measurements with the micro-flow flow generator using syringes of $50 \mathrm{ml}, 10 \mathrm{ml}$ and $1 \mathrm{ml}$ volume on the micro-flow facility. the case where water is used as calibration liquid. Therefore the first calibration measurements with oil are performed without a DUT to limit pressure drop.

The calibration results using the three reference oils are consistent with the results where water is used as calibration liquid, as can be seen in Fig. 14. A slight viscosity-dependent trend can be observed and needs further investigation.

These consistent calibration results allow the flow generator to be used as a transfer standard and to perform the measurements according to the scheme shown in Fig. 15, where the liquid is pressed with the METAS flow generator through the DUT and into the waste.

\section{Validation of the Milli-flow Facility}

It is imperative in metrological practice to verify the stated measurement uncertainties which are defined according to the Guide to the Expression of Uncertainty in Measurement ${ }^{77}$ for the whole flow rate range. This is done by means of an intercomparison with other flow facilities from several National Metrology Institutes (NMIs). A well-characterized and very

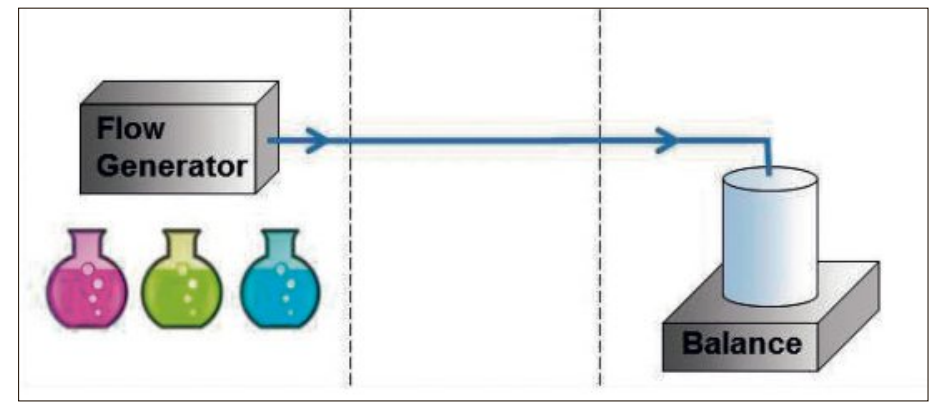

Fig. 13. Calibration of the milli-flow flow generator with other liquids by means of the dynamic gravimetric method.

stable flow device is used as a transfer standard. All participants calibrate this flow device. These intercomparisons are organized within the regional metrology organizations (RMOs), ${ }^{[8]}$ and one of the main roles is to support mutual confidence in calibration and measurement capabilities (CMCs) of the participating NMIs (CIPM-MRA). [9] The validated CMCs, which consist of the flow rate range with the corresponding uncertainties, are then published in the BIPM key comparison database. ${ }^{[10]}$ To validate this milli-flow facility we participated in the intercomparison EURAMET project 1379.[11]

To evaluate all the results, the reference value and its standard uncertainty are determined according to the procedure published by Cox. ${ }^{[12,13]}$ The reference value labeled as ' $\mathrm{RV}$ ' and the calibration results of all the participants are shown in Fig. 16. Most of these calibration results are in agreement with the reference value. However, detailed discussion is beyond the scope of this article and can be read in the report of EURAMET project 1379. [11]

\section{Conclusion}

To address the issue of calibrating flow devices using process-oriented liquids METAS has developed a milli-flow facility for flow rates from $0.2 \mathrm{ml} / \mathrm{min}$ to $400 \mathrm{ml} /$

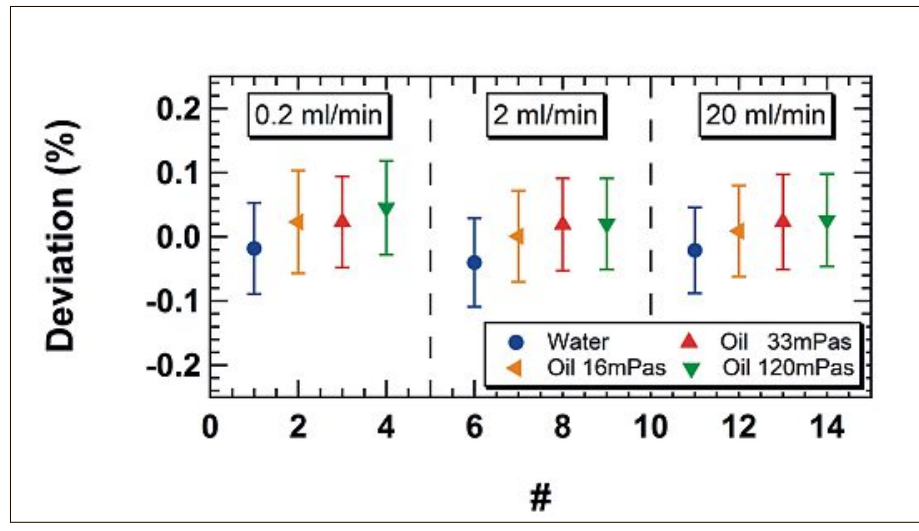

Fig. 14. Calibration of the milli-flow flow generator with a syringe of $10 \mathrm{ml}$ volume and other liquids by means of the dynamic gravimetric method.

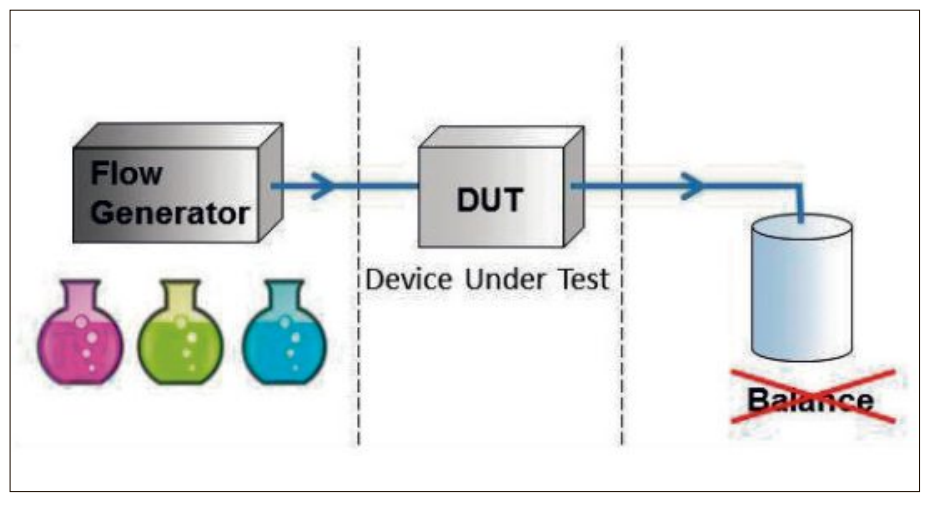

Fig. 15. Working principle of the calibrated milli-flow flow generator without collecting the liquid on the balance. The flow generator pumps any liquid through the DUT and into waste. 
Fig. 16. Intercomparison EURAMET project 1379. The calibration results of a wellcharacterized flow device obtained by all the participants and the reference value labeled as 'RV'.

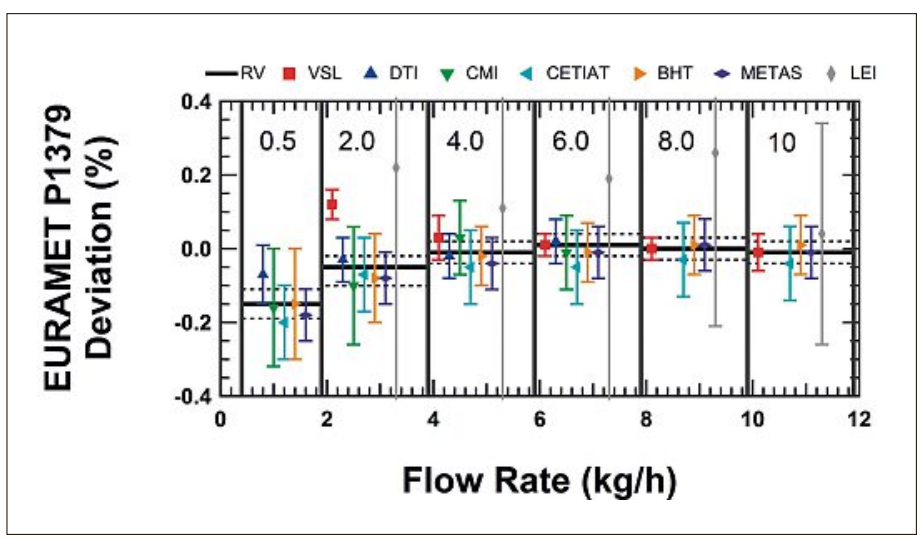

min with an uncertainty of $0.07 \%$ with a METAS flow generator allowing measurements with any liquid other than water. Moreover, a METAS micro-flow flow generator has been built for the flow rate range from $50 \mathrm{nl} / \mathrm{min}$ to $5 \mathrm{ml} / \mathrm{min}$ with uncertainties from $0.9 \%$ to $0.1 \%$. Traceability is guaranteed through the calibration of the generated flow rates of the METAS flow generator by means of the dynamic gravimetric method where a liquid of wellknown density and a well-controlled evaporation rate is used. As the METAS flow
[1] H. Bissig, M. Tschannen, M. de Huu, Flow Meas. and Instrum. 2015, 44, 34

[2] H. Bissig, H. T. Petter, P. Lucas, E. Batista E. Filipe, N. Almeida, L. F. Ribeiro, J. Gala, R. Martins, B. Savanier, F. Ogheard, A. K. Niemann, J. Lötters, W. Sparreboom, Biomed Eng-Biomed Tech 2015, 60, 301.

[3] BIPM, the BIPM key comparison database, internal NMI service identifier 5135-CH11, http://kcdb.bipm.org/appendixC/

[4] K-H. Cheong, R. Doihara, T. Shimada, Y. Terao, 'Expansion of Calibration Flow Range of Small Liquid Hydrocarbon Flow Facility at NMIJ', in Proc. ISFFM, 2015.

[5] BIPM, the BIPM key comparison database, internal NMI service identifier UK1, http://kcdb. bipm.org/appendixC/

[6] Euroloop: test facility for high pressure and high flow gas and liquid meters, http://nmieuroloop.nl/

[7] http://www.iso.org/sites/JCGM/GUMintroduction.htm

[8] BIPM. Regional metrology organizations (RMOs) recognized within the framework of the CIPM MRA. Available at www.bipm.org/en/ worldwide-metrology/regional/

[9] BIPM. International equivalence of measurements: the CIPM MRA. Available at http:// www.bipm.org/en/cipm-mra/

[10] BIPM. The BIPM key comparison database. Available at $h t t p: / / k c d b . b i p m . o r g /$

Received: January 17, 2018 [11] https://www.euramet.org/technical-committees/ search-tc-projects/

[12] M. G. Cox, Metrologia 2002, 39, 589.

[13] M. G. Cox, Metrologia 2007, 44, 187. 